ESTRATEGIAS DE NEGOCIO DE LAS PYMES FAMILIARESEN CIUDADVICTORIA, TAMAULIPAS-MÉXICO ${ }^{1}$

\title{
BUSINESS STRATEGIES OF FAMILY SMES IN VICTORIA CITY, TAMAULIPAS-MEXICO
}

\author{
Mariana Zerón Félix ${ }^{2}$, Yesenia Sánchez Tovar ${ }^{3}$ \& Nallely Guadalupe Hernández ${ }^{4}$ \\ Universidad Tamaulipas, México
}

RECIBIDO: Febrero 18 de 2015

\section{RESUMEN}

El objetivo del presente artículo de investigación consistió en identificar la tipología de estrategias de negocios que implementan las pymes con estructura familiar en Ciudad Victoria, Tamaulipas, México. Se desarrolló a partir de un enfoque cuantitativo de tipo descriptivo, con diseño no experimental transversal, en el que se contrastaron dos enfoques de tipologías de estrategias, resultando indicada, considerando el sector económico objeto de estudio y a la unidad de análisis, la tipología de Miles y Snow (1978). La población estuvo conformada por 210 empresas clasificadas como pymes. Se utilizó para recolección de datos la encuesta desarrollada por Aragón-Correa (1996), aplicado a una muestra de 73 pymes familiares del sector comercial, con error de $\pm 8.35 \%$ y un nivel de confianza del $95 \%$. Y a través de la realización de un análisis multivariante clúster utilizando el método de k-medias, se obtuvo como resultado que un $32.88 \%$ de las empresas en Ciudad Victoria optan por una estrategia reactiva y un 31.50\%, por una defensiva, denotando un estilo conservador. Se concluyó que las pymes con estructura familiar de Ciudad Victoria son vulnerables debido a que en su mayoría no generan un plan estratégico, lo cual provoca la dificultad en la creación de capacidades y atributos necesarios que respondan ante los acontecimientos del entorno dinámico y competitivo actual.

Palabras clave: Competitividad, Estrategia de negocio, Pymes familiares.

\begin{abstract}
The objective of this research paper is to identify the type of business strategies that implement the SMEs with a family structure in the city of Victoria, Tamaulipas, Mexico. It was developed from a quantitative descriptive approach, with a non-experimental transverse design, where two approaches of different types of strategies were compared, resulting appropriate, considering the economic sector under study and the unit of analysis, the typology of Miles and Snow (1978). The population consisted of 210 companies classified as SMEs. The survey made for data collection was developed by Aragon-Correa (1996), applied to a sample of 73 SMEs which were familiar to the commercial sector, with an error of $\pm 8.35 \%$ and a confidence level of $95 \%$. And through carrying out a multivariate analysis using the method of cluster k-means, it was obtained a result that indicates that $32.88 \%$ of businesses in the town of Victoria opt for a reactive strategy and $31.50 \%$ for a defensive one, thus showing a conservative style. It was concluded that the SMEs with a family structure in the city of Victoria are vulnerable because most of them do not generate a strategic plan. This causes great difficulty in generating the capabilities and necessary attributes that respond to the dynamic events of today's competitive environment.
\end{abstract}

Keywords: Competitiveness, Business strategy, Family SMEs.

Este artículo se puede referenciar

Zerón. M., Sánchez, Y., \& Hernández, N. (2015). Estrategias de Negocio de las Pymes familiares en Ciudad Victoria, Tamaulipas (México). En Desarrollo Gerencial. Revista de la facultad de ciencias económicas, administrativas y contables. 7-2, Universidad Simón Bolívar, Barranquilla, Colombia, Pp.19-35. http://dx.doi.org/10.17081/dege.7.2.1178

\footnotetext{
${ }^{1}$ Proyecto de investigación. Estrategias de negocio de las pymes familiares en ciudad Victoria, Tamaulipas, México, la cual fue financiada por la Universidad Autónoma de Tamaulipas, durante el año 2014.

${ }^{2}$ Doctor por la Universidad Autónoma de Tamaulipas (UAT). Responsable de la División de Posgrado e Investigación, Profesor e investigador de Tiempo Completo de la Facultad de Comercio y Administración Victoria de la UAT. Email: zfmariana@uat.edu.mx.

${ }^{3}$ Doctor en Economía y Gestión de las Organizaciones por la Universidad de Zaragoza. Coordinador del Doctorado en Ciencias Administrativas y Profesor e investigador de Tiempo Completo de la Facultad de Comercio y Administración Victoria. Miembro del Sistema Nacional de Investigadores.Email: yesanchez@uat.edu.mx.

${ }^{4}$ Maestro en Dirección Empresarial por la Universidad Autónoma de Tamaulipas. Profesor e investigador de Tiempo Completo de la Facultad de Comercio y Administración Victoria.Email:nghernandez@uat.edu.mx
}

Desarrollo Gerencial, 7 (2) Pp. 19 - 35 Julio-Diciembre 2015. ISSN: 2145-5147 (On Line). Universidad Simón Bolívar. Barranquilla-Colombia. Contactos: desarrollogerencial@unisimonbolivar.edu.co 


\section{1.- INTRODUCCIÓN}

En la actualidad, las pequeñas y medianas empresas (pymes) desempeñan un papel fundamental en la economía a nivel internacional debido a que se les ha reconocido como una fuente importante de generación de empleos, al igual que por su participación en el comercio exterior, por ser desarrolladoras de capital humano, así como por fungir como distribuidoras de la riqueza, teniendo una destacable participación en el PIB de las naciones (Cubillo, 1997). Ponderando también que este tipo de empresas representan un porcentaje considerable de las unidades económicas a nivel mundial (Instituto Nacional de Estadística y Geografía [INEGI], 2009).

Este breve panorama a nivel internacional nos permite, de tal forma, destacar que la base productiva nacional de México está ampliamente representada por el sector de las pymes, al ser estas el 98.4\% del total de las unidades económicas del sector servicios, comercio y manufactura en el país. Así mismo, las pymes sostienen el 72\% de la población económicamente activa y amparan al 90.2\% del personal ocupado (INEGI, 2009). En consecuencia, Millán (2011) enfatiza, basándose en información del Banco Mundial, que las pymes en México contribuyen en un 52\% al producto interno bruto (PIB).

Pero en contraparte, como menciona Porter (2002), "las empresas en todo el mundo han visto disminuir su crecimiento y enfrentar a competidores nacionales e internacionales” (pp. XVII). Es así que a pesar de la contribución significativa que representan las pymes para la economía de México, estas no han alcanzado un nivel competitivo como para posicionarse en el mercado global, puesto que la tasa de mortalidad de estas empresas pone de manifiesto su bajo nivel de desempeño, destacando que de las 200 mil empresas que anualmente abren sus puertas en México cada año, solamente 35 mil sobreviven dos años más tarde (Tan, López-Acevedo, Flores, Rubio, Slota, Tinajero y Beker, 2007; Gómez, 2006).

Por lo tanto, la subsistencia de las pequeñas y medianas empresas (pymes) es probablemente una de las tareas más difíciles para un empresario (Palomo, 2005; Kauffman, 2001; Sánchez, 2003), por lo que surge la necesidad de adecuar sus estrategias, sus recursos (humanos, organizacionales, financieros, tecnológicos), sus productos y principalmente su actitud ante el entorno complejo y dinámico en el que operan actualmente; trayendo consigo la posibilidad de mejorar su nivel competitivo (García, 2007; Cermeli, 2013; Armenteros, Elizondo, Ballesteros y Molina, 2012).

En consecuencia, en años recientes la literatura se ha abocado a desarrollar un mayor número de investigaciones orientadas a explicar y analizar la relación entre el uso de diferentes técnicas de dirección, estrategias o estructuras organizativas con el rendimiento financiero o éxito de la empresa (Maranto, 2003; Ballina, Medina y Martínez, 2006; Marroquín, 2008; Aguilera y González, 2012). 
En relación con este tema, estudios como los realizados por Aragón y Rubio (2005); Cermeli (2013); Sánchez, Zerón y Mendoza (2014) sostienen que la estrategia empresarial existente en cualquier tipo de organización es uno los elementos que presenta mayor relación con el desempeño y consecuente éxito de la misma, debido a que la estrategia es la que indica los principios y rutas fundamentales que orientarán el proceso administrativo que va a permitir alcanzar los objetivos a los que desea llegar una organización.

Por lo que la presente investigación plantea, en primera instancia, identificar principalmente la tipología de estrategias empresariales que implementan las pymes con estructura familiar en Ciudad Victoria, Tamaulipas.

Lo anterior se llevó a cabo desde la visualización de la tipología de Milles y Snow (1978), para conocer cómo las pymes con estructura familiar de Ciudad Victoria encaminan sus esfuerzos, la aplicación de sus recursos, ya sea dentro de un enfoque de estrategia exploradora, analizadora, reactiva o defensiva. Una vez que se conoce qué tipo de estrategia es utilizada por la empresa que forma parte de la unidad de análisis, se debe determinar la relación existente entra la estrategia y la competitividad, contestando de este modo nuestra pregunta de investigación: ¿qué estrategias de negocio implementan las pymes familiares en Cd. Victoria, Tamaulipas, México?

\section{Tipología de estrategia}

Es necesario precisar las tipologías de estrategias empresariales que son pautas de análisis al comportamiento estratégico de las organizaciones, de tal modo que para concebir la presente investigación, así como para dar respuesta a nuestra pregunta de investigación, es necesario abordar la tipología que para dicho estudio se consideró apropiada y el porqué de su uso.

\section{$\checkmark$ Genéricas de Michel Porter (1980)}

En sus obras, Porter (1980, 1990 y 2002) reconoce el impacto de las decisiones directivas en la estrategia de la empresa y sostiene que una empresa puede, mediante la elección de su estrategia, alcanzar una posición competitiva dentro de la industria que le genere altas tasas de retorno, aun cuando la rentabilidad promedio de la industria sea modesta. Las actividades de pensamiento analítico de la organización determinan racionalmente cómo la empresa se posiciona estratégicamente.

Las estrategias genéricas de Porter (1980) han sido aceptadas como una opción muy efectiva para impulsar la generación de la ventaja competitiva (Camisón, Garrigos y Palacios, 2007). No obstante, autores como Hambrick y Lei (1985) argumentan con lógica que la estructura y naturaleza de la industria puede favorecer en mayor o menor medida a alguna de estas estrategias, por adaptarse de mejor forma a las características del mercado a explotar. Así, para Castro (2010) las acciones específicas requeridas para 
la implementación de alguna de las estrategias propuestas en el modelo de Porter (1980) difieren ampliamente en cada sector.

Sin embargo, se debe precisar que esta tipología ha sido mayormente aplicada al estudio de empresas pertenecientes al sector industrial, dejando un vacío en el estudio del posicionamiento estratégico de las empresas que se desenvuelven en los otros sectores económicos.

\section{$\checkmark$ Directivas de Milles y Snow (1978)}

Por otro lado, una de las tipologías de mayor reconocimiento a nivel global es precisamente la de los autores Miles y Snow (1978). El argumento que sustenta esta afirmación es el gran número de trabajos científicos (Zahra y Pearce II, 1990; Dvir, Segev y Shenhar, 1993; Hambrick, 1982; Hambrick, 1983c; Conan, Mokwa y Varadarajan, 1990; Shortell y Zajac, 1990; Segev y Weber, 1991; Beekum y Ginn, 1993; Veliyath y Shortell, 1993; Aragón, 1996; y Sánchez, Zerón y Mendoza, 2014) que como puede comprobarse basan, en mayor o menor grado, su desarrollo en dicha propuesta de la clasificación de la estrategia empresarial.

Al adentrarse en la tipología propuesta por Miles y Snow (1978), se puede observar que estos autores sostienen que la efectividad de una empresa está vinculada con la labor desempeñada por sus directivos, en lo referente a las decisiones concretadas ante tres tipos de problemas del entorno: el problema empresarial, el problema de tecnología y el problema administrativo.

El conocimiento de estas tres dimensiones, de acuerdo con Miles y Snow (1978), permite a las empresas dar una respuesta efectiva al ciclo adaptivo del entorno a través de cuatro estrategias o patrones estratégicos básicos: exploradora, analizadora, defensiva y reactiva.

Los tres primeros tipos de estrategias (exploradoras, defensoras y analizadoras) tienden a ser constantes en la elección estratégica y muestran un buen desempeño, por lo que se pueden considerar estrategias de éxito. Por el contrario, la estrategia reactiva está abocada al fracaso, dado que no responde a un patrón estratégico planificado debido a que responde de manera inadecuada a las presiones ambientales que se le van presentando y, en este sentido, se puede considerar incluso como una "no estrategia" (Desarbo, Di Benedetto, Song y Sinha, 2005; Sánchez, Zerón y Mendoza, 2014).

Desde el punto de vista de McDaniel y Kolari (1987), el modelo propuesto por Miles y Snow (1978) se encuentra entre los que más resonancia han alcanzado gracias a su concepción de la organización como un sistema complejo e integrado en interacción con su entorno. Para algunos autores (Segev, 1989; Walker y Reukert, 1987; Austin, Trimm y Sobczak, 1995; Lado, 1997) la utilidad de la tipología de estrategia de Miles y Snow (1978) versa sobre la facilidad que brinda para reducir el enorme número de variables que 
demandan la atención de los directivos a un conjunto más pequeño de factores, lo que hace más simple la evaluación de las variables del entorno y de las variables relativas a la implementación de la estrategia.

En consecuencia, la presente investigación utiliza la tipología de Miles y Snow (1978), justificándose en la descripción de la pyme como una organización cambiante al enfrentarse a un entorno altamente competitivo. Además de que esta tipología permite analizar de forma más precisa el comportamiento estratégico de las empresas en una mayor diversidad de sectores económicos, como lo son el comercial y el de servicios, lo que en la actualidad es de gran importancia debido a que las economías se caracterizan por ser muy variadas en lo relativo a los sectores que las estructuran.

\section{2.- MÉTODO}

\section{Diseño}

Esta investigación se elaboró con un enfoque cuantitativo, puesto que la recolección de datos se realizó con base en una medición numérica de la variable Estrategia a través de un análisis estadístico que permitiera establecer los patrones de comportamiento estratégico de las empresas (Bernal, 2010). Teniendo un alcance descriptivo debido a que busca identificar características y perfiles estratégicos de las empresas (Hernández, Fernández y Baptista, 2006), y un diseño no experimental, de acuerdo con Münch y Ángeles (2007), puesto que no se manipularon las variables objeto de estudio, solo se observó la realidad a partir de la recolección de datos, esto sin pretender algún cambio de la misma. Así mismo, es un estudio transversal (Hernández, Fernández y Baptista, 2006), ya que solamente se estudiaron las estrategias implementadas por las pymes con estructura familiar en Ciudad Victoria, Tamaulipas, en un periodo determinado.

\section{Participantes}

La población objeto de estudio estuvo conformada por las pymes con actividad comercial o de servicios constituidas legalmente en Ciudad Victoria, Tamaulipas, al norte de México. Las pymes son clasificadas en función del número de empleados. De acuerdo con el Diario Oficial de la Federación (2013, 28 diciembre), se establece que las pequeñas empresas para el sector comercial y de servicios serán aquellas con un número de trabajadores de 11 a 50, mientras que las medianas empresas de dichos sectores serán aquellas que tienen de 51 a 100 empleados. Desde esta clasificación, en Ciudad Victoria existen 210 empresas clasificadas como pymes, las cuales constituyen el universo o total de la población. 
En este sentido, el tamaño de la población fue obtenido a partir de las empresas que cumplen la característica de tamaño y que se encuentran registradas en el Directorio Estadístico Nacional de Unidades Económicas (DENUE) del INEGI (2014). Una vez conocido el universo, se determinó la muestra a través de un muestreo estadístico no probabilístico por conveniencia, debido a que las empresas fueron elegidas a juicio de los investigadores (Hernández, Fernández y Baptista, 2006) considerando aquellas que se encontraran en la zona central de la ciudad por ser un punto de fácil acceso debido a la distribución geográfica de la ciudad. Para determinar la muestra se consideró un error de $\pm 8.35 \%$ y un nivel de confianza del 95\%. La muestra obtenida fue de 73 empresas.

\section{Instrumentos}

El cuestionario aplicado fue desarrollado por Aragón-Correa (1996) basado en la tipología de Miles y Snow (1978). Dicho instrumento integrado por 18 ítems recoge las tres dimensiones que integran la estrategia empresarial. El nivel de cada uno de los ítems de la escala permite definir si se trata de una empresa con estrategia exploradora, defensiva, analizadora o reactiva. El cuestionario además recoge datos socioeconómicos de la empresa como la edad, tipo de estructura, tamaño y ratios de crecimiento financiero, entre otros. Dicho cuestionario fue validado en su contenido por ocho investigadores y profesionales del área de estrategia de la Universidad Autónoma de Tamaulipas, logrando adaptarlo al empresario de la región.

La recolección de datos para el estudio estuvo centrada en la aplicación de este cuestionario a una muestra representativa, logrando validar 84 cuestionarios. La recogida de la información se llevó a cabo en los meses de octubre a diciembre de 2013. Cabe mencionar que de los 84 cuestionarios aplicados, solamente 73 correspondieron a pymes con estructura familiar, por lo que, para efectos de esta investigación, las 73 empresas son la muestra para el análisis de la tipología de estrategias.

\section{Procedimientos}

El presente estudio forma parte de un proyecto de investigación. Se realizó en las siguientes fases: identificación y análisis de la literatura existente sobre los modelos de estrategias identificando dos: el de Miles y Snow y el de Michael Porter.

Se seleccionó el modelo de Miles y Snow (1978), que propone cuatro tipos de estrategias empresariales: exploradora, analizadora, defensiva y reactiva. Este modelo recoge una perspectiva voluntarista de la estrategia que integra estructura, estrategia y procesos; las cuales se convierten en modelos que sirven de base para la toma de decisiones empresariales a seguir. 
Se aplicó, a las 73 pymes, un instrumento a través de la técnica de investigación de la encuesta, la cual fue desarrollada por Aragón-Correa (1996), basado en la tipología de Miles y Snow (1978).

Se realizó el vaciado a una base de datos de las respuestas de los gerentes de las pymes incluidas en las encuestas, para su posterior análisis estadístico, en el paquete estadístico para las Ciencias Sociales SPSS versión 20 .

Se estructuró el marco teórico con base en la revisión de literatura y, finalmente, se redactó el artículo.

\section{3.- RESULTADOS}

El tipo de resultado es analítico, para la identificación de la tipología de estrategias de negocio de las pymes familiares en Ciudad Victoria (Tamaulipas, México); de la muestra: son 73 empresas de corte familiar, tal como se muestra en la Gráfica 1, por lo que el análisis de la tipología de estrategia se analizará a partir del $87 \%$ de las empresas encuestadas. Así mismo, se puede observar en la Tabla 1 que el $58 \%$ de las pymes corresponden al sector comercial. Cabe mencionar que la economía de Ciudad Victoria está basada en el sector terciario, puesto que no es una ciudad industrial ni desarrolla actividades primarias, por lo que dos tercios del valor agregado económico que se genera en la ciudad proviene del comercio y los servicios, esto de acuerdo con los datos de INEGI (2009). En este sentido, el sector comercial reporta aproximadamente 2.360 .032 millones de pesos de valor económico agregado a la ciudad, de los 8.644.800 millones de pesos que se generan por el total de actividades, siendo así el sector comercial el sustento principal de la ciudad.

Gráfica 1. Pymes encuestadas

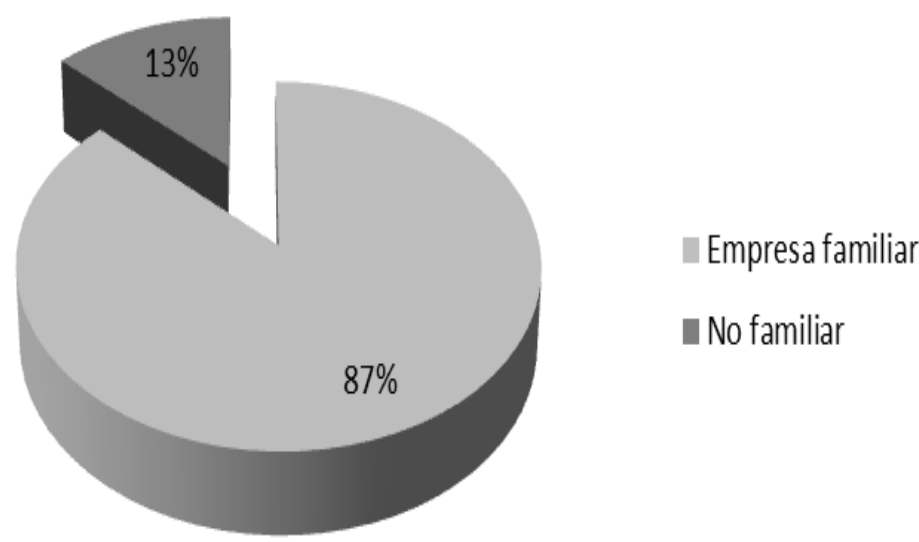

Fuente: Elaboración propia 2013-2014 
Tabla 1. Generalidades de las empresas encuestadas

\begin{tabular}{|c|c|c|}
\hline Variable & Descripción & Porcentaje \\
\hline \multirow{2}{*}{ Sector productivo } & Servicios & $42 \%$ \\
& Comercial & $58 \%$ \\
\hline Tipo de pyme & Familiar & $87 \%$ \\
& No familiar & $13 \%$ \\
\hline
\end{tabular}

Fuente: Elaboración propia 2013-2014

En relación con las generalidades del encuestado en la Tabla 2 se destacan datos como la edad, el sexo y la escolaridad; acentuando que el 65\% se encuentra entre los rangos de 41 a 50 años y más de 50 años de edad, el $93 \%$ cuenta con estudios universitarios o posgrado y en el $78 \%$ de los casos son hombres los que dirigen las empresas. Las cifras anteriores sugieren un considerable nivel de experiencia y preparación de los directivos de las pymes victorenses, aspecto de suma importancia debido a que puede suponer una fortaleza de estas empresas para hacer frente a los retos presentes en la economía actual.

Tabla 2. Generalidades del encuestado

\begin{tabular}{|l|l|c|}
\hline \multicolumn{1}{|c|}{ Variable } & \multicolumn{1}{|c|}{ Descripción } & Porcentaje \\
\hline Edad & Menor de 20 & $2 \%$ \\
& De 21 a 30 & $11 \%$ \\
& De 31 a 40 & $21 \%$ \\
& De 41 a 50 & $32 \%$ \\
& Más de 50 años & $33 \%$ \\
\hline Escolaridad & Primaria & $1 \%$ \\
& Secundaria & $1 \%$ \\
& Bachillerato & $3 \%$ \\
& Técnico & $2 \%$ \\
& Universitario & $77 \%$ \\
& Posgrado & $16 \%$ \\
\hline Sexo & Hombre & $78 \%$ \\
& Mujer & $22 \%$ \\
\hline
\end{tabular}

Fuente: Elaboración propia 2013 - 2014.

De las 73 empresas familiares, el 53\% si realiza un plan mientras que el 47\% no lo realiza (Gráfica 2). Este resultado concuerda con lo establecido por Kauffman (2001) quien señala que uno de los principales problemas de las pymes es la carencia de sistemas de planificación empresarial. 
Gráfica 2: Pymes familiares que realizan un plan estratégico

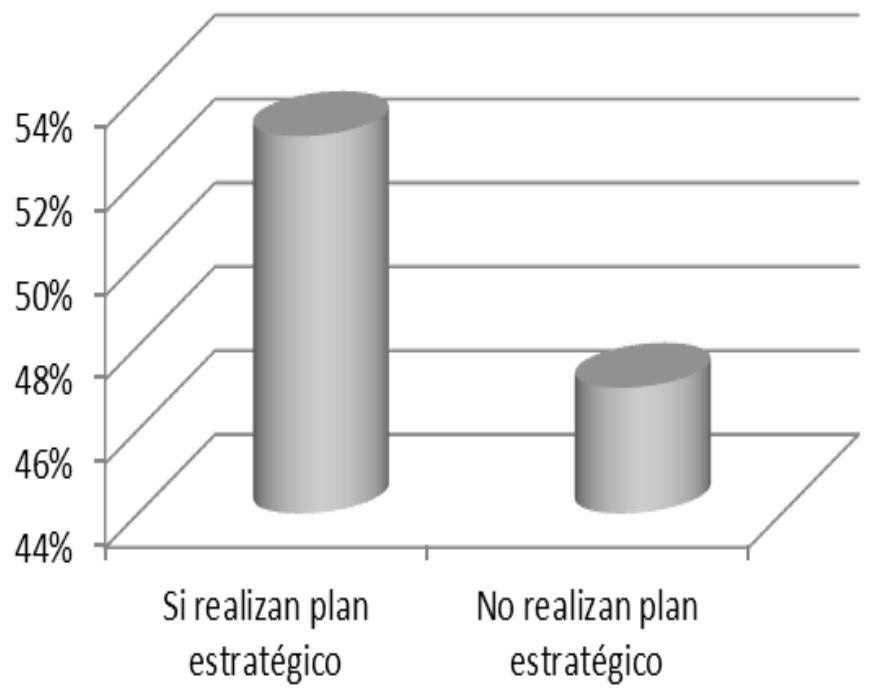

Fuente. Elaboración propia 2013 - 2014.

Con el objetivo de identificar el tipo de estrategia empresarial (exploradora, analizadora, defensiva y reactiva) aplicada por cada una de las empresas observadas, se realizó un análisis multivariante clúster utilizando el método de k-medias. Dicho método es considerado de carácter no estadístico y permite reducir una compleja cantidad de datos en grupos en los que los miembros comparten características similares. En esta investigación cada una de las empresas fue agrupada en uno de los cuatro conglomerados que corresponden a las cuatro estrategias definidas por Miles y Snow (1978).

En este sentido, cabe precisar que las empresas exploradoras se caracterizan por la búsqueda continua de nuevas oportunidades. Se las considera creadoras de cambio, sin embargo, su dedicación por innovar las hace no del todo eficientes. Por su parte, las empresas analizadoras se distinguen por desenvolverse tanto en un entorno estable, en el que se vuelven rutinarias y eficientes, como en uno cambiante en el que los directivos vigilan permanentemente a los competidores y adoptan en el menor tiempo las ideas que más prometen (Miles y Snow, 1978).

En el caso de las empresas reactivas, estas se diferencian por carecer de una relación entre estrategia y estructura, lo que deriva en que sus respuestas al cambio e incertidumbre del entorno se den por la presión generada por dichas situaciones. Por último, las empresas defensivas se distinguen por contar con una alta dirección que no busca nuevas oportunidades fuera de su negocio y, por tanto, se mantienen en un campo de mercado estrecho. Tal como se muestra en la Tabla 3. 
Tabla 3. Puntuaciones medias de variables por grupo

\begin{tabular}{|l|c|c|c|c|}
\hline \multicolumn{1}{|c|}{ Ítem } & \multicolumn{3}{c|}{ Conglomerado } \\
\cline { 2 - 5 } & Grupo 1 & Grupo 2 & Grupo 3 & Grupo 4 \\
\hline Desarrollo nuevos productos & 4.73684 & 4.44444 & 3.75000 & 2.04348 \\
Análisis del entorno & 5.94737 & 3.50000 & 4.70833 & 3.52174 \\
Forma de competir en el mercado & 2.42105 & 5.33333 & 2.54167 & 3.08696 \\
Planeación del crecimiento en el mercado & 5.36842 & 4.88889 & 2.62500 & 2.69565 \\
Velocidad de reacción al entorno & 2.36842 & 4.33333 & 2.08333 & 4.30435 \\
Tipo de diversificación & 1.89474 & 3.94444 & 2.08333 & 2.43478 \\
Proceso tecnológico & 5.84211 & 3.55556 & 5.08333 & 1.95652 \\
Núcleo tecnológico & 5.68421 & 4.16667 & 4.58333 & 3.91304 \\
Nivel tecnológico frente al mercado & 6.00000 & 3.77778 & 2.08333 & 2.65217 \\
Áreas de influencia de la empresa & 5.47368 & 2.61111 & 2.54167 & 1.69565 \\
Especialización de la mano de obra & 6.26316 & 4.33333 & 4.66667 & 3.26087 \\
Nivel de estructuración jerárquica & 1.73684 & 3.44444 & 3.04167 & 2.34783 \\
Medición del rendimiento organizacional & 2.94737 & 4.00000 & 2.50000 & 1.95652 \\
Estabilidad de la dirección del negocio & 5.31579 & 2.44444 & 2.04167 & 3.00000 \\
MEDIA & 4.38596 & 3.81852 & 3.31111 & 2.84058 \\
VARIANZA & 2.605971 & 0.66192 & 1.323349 & 0.586683 \\
Tipo de estrategia & Explorador & Analizador & Reactivo & Defensivo \\
\hline
\end{tabular}

Fuente: Elaboración propia con base en el programa Statistical Packageforthe Social Sciences (SPSS) versión 20.0.

En primer lugar, la Tabla 3, expuesta anteriormente, muestra los valores finales de los centros de los conglomerados. Dichos valores representan las medias de cada variable en cada conglomerado final, por lo que reflejan los atributos de cada uno de estos. En este sentido, se observa que el conglomerado 1 contiene los valores más altos de medias para las variables, por lo que corresponde a la estrategia exploradora. El conglomerado 4 muestra los valores de variables más bajos representando la estrategia defensiva. El conglomerado 3 refleja a la estrategia reactiva y el conglomerado 2 se refiere a la estrategia analizadora. Por su parte, en la Tabla 4 presenta un resumen del número de empresas familiares (73) agrupadas en cada estrategia, identificando 19 empresas exploradoras, 18 empresas analizadoras, 24 reactivas y 23 defensivas.

Tabla 4. Resumen de casos por conglomerados

\begin{tabular}{|c|l|c|}
\hline Conglomerado & \multicolumn{1}{|c|}{ Clasificación estratégica } & Número de empresas \\
\hline Grupo 1 & Estrategia exploradora & 12 \\
\hline Grupo 2 & Estrategia analizadora & 14 \\
\hline Grupo 3 & Estrategia reactiva & 24 \\
\hline Grupo 4 & Estrategia defensiva & 23 \\
\hline Casos válidos & & 73 \\
\hline Caso perdidos & & 0 \\
\hline
\end{tabular}

Fuente: Elaboración propia 2013 - 2014. 
En síntesis, las empresas victorenses familiares siguen, en su mayoría, una estrategia reactiva y defensiva ya que representan el $64.33 \%$ del total de empresas de estructura familiar (Gráfica 3).

Gráficaa 3: Clasificación de empresas familiares de acuerdo con su estrategia

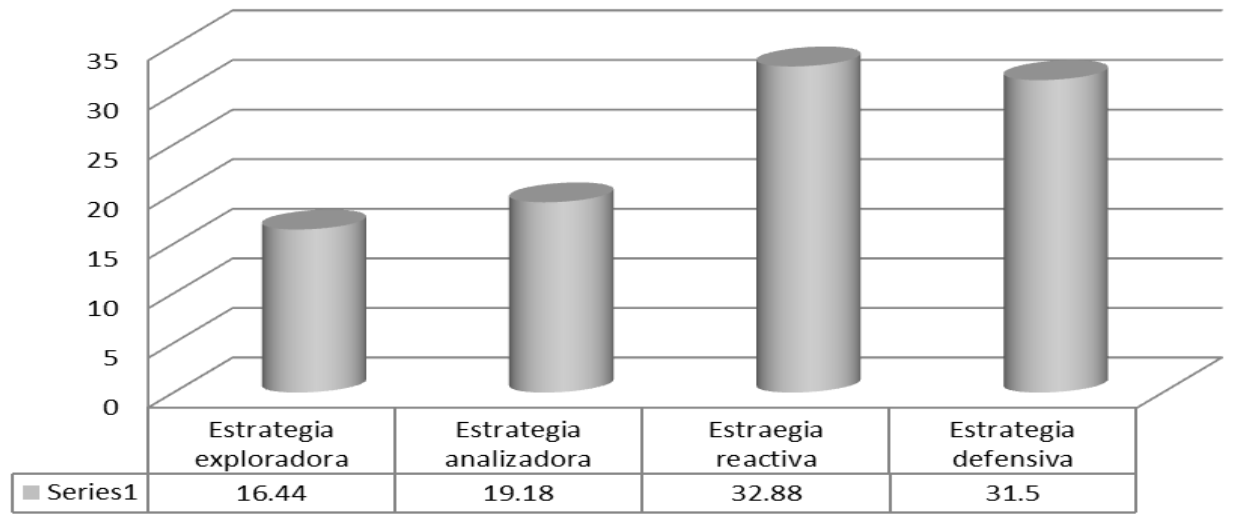

Fuente: Elaboración propia 2013 - 2014.

\section{4.- DISCUSIÓN}

La estrategia empresarial ha sido analizada por la literatura como una de las causas de la ventaja competitiva que permite a una empresa obtener el éxito de manera superior al resto de las empresas que participan en un mismo sector (Lippman y Rumelt, 1982; Reed y DeFilippi, 1990; Grant, 1991; Cockburn, Henderson y Stern, 2000).

Por otro lado, el hecho de estudiar la empresa familiar radica en los dos rasgos característicos de este tipo de empresa: las relaciones de parentesco, vinculadas a la descendencia, y las relaciones de afecto y de pertenencia entre los miembros (Alberdi y López, 1995; Shanker y Astrachan, 1996). Esto permite suponer que el comportamiento estratégico de este tipo de empresas se puede ver afectado por estos rasgos. Lo anterior justifica, en base a los hallazgos, establecer que a este tipo de empresas de la localidad se les dificulte coordinar eficientemente los recursos físicos y humanos, provocando el retraso de su crecimiento.

Lo anterior causa contradicción dentro de lo observado en la tipología de Miles y Snow (1978), observando la dirección de las organizaciones desde la perspectiva voluntarista de la estrategia que integra estructura, estrategia y procesos; las cuales se convierten en modelos que sirven de base para la toma de decisiones empresariales.

La no existencia de una estrategia también corresponde a la existencia de una estrategia reactiva, como lo explican Miles y Snow (1978). Así, se observa que la mayoría de las empresas familiares siguen el 
patrón de una estrategia reactiva: son empresas a las que se les dificulta concluir alguna acción, no tienen metas a largo plazo y se ajustan a las situaciones conforme se ven forzadas por las presiones ambientales. Son empresas que no cuentan con un sistema de control adecuado y se encuentran en total inestabilidad.

Esto coincide, en cierta medida, con los resultados encontrados en esta investigación, puesto que Cd. Victoria es una ciudad que se desarrolla alrededor del aparato de gobierno y de la universidad del Estado, al ser capital de Tamaulipas, teniendo poca industria y por tanto poco ambiente de desarrollo competitivo, lo que da a las empresas cierta comodidad que se refleja, en su mayoría, en una estrategia defensiva y reactiva.

Otra de las estrategias usuales en Ciudad Victoria, por parte de las empresas familiares, es la estrategia defensiva, es decir, intentan mantener un lugar seguro en un área estable para su producto en lugar de concentrarse en un nuevo producto o tratar de desarrollarse dentro de nuevos mercados; por lo tanto, permanecen dentro de una gama de productos limitada, centrándose más en la eficacia de la utilización de los recursos y las mejoras del proceso, con el objeto de disminuir costos.

Estos resultados, a su vez, son soportados por las teorías de la empresa familiar desarrolladas por autores como Daily y Dollinger (1991) y Van Gils, Voordecker y Van den Heuvel (2004), quienes deducen que las empresas familiares tienden a seguir estrategias de carácter defensivo y reactivo puesto que buscan mantener el control en base a una reducción de costos, lo cual es una característica de las estrategias de corte defensivo. Estas aseveraciones teóricas son soportadas en esta investigación, puesto que los resultados reflejan que las empresas familiares de Ciudad Victoria tienden a este tipo de estrategias.

En el mismo sentido, el cambio de estrategias de acuerdo a la teoría de la empresa familiar deberá darse conforme la dirección sea atendida por personas externas a la familia (McCann, León-Guerrero y Haley, 2001), lo cual también se puede presumir en esta investigación, puesto que se nota, a su vez, que en menor medida, pero de forma creciente las empresas están intentando modificar sus planes de conducta organizacional dirigiéndose a una estrategia analizadora, la que se caracteriza por buscar un equilibrio entre los costos y la eficiencia, y la innovación y tomar riesgos.

Otros estudios hechos en México (Aragón et al., 2010) ponen de manifiesto que el tipo de estrategia que debe seguirse depende de la región, reflejándose que para el sur de México las Pyme optan por estrategias más proactivas como la exploradora y la analizadora para alcanzar el éxito. A su vez este mismo estudio destaca que las pyme no se ven influenciadas por la formación de los directores al momento de elegir una estrategia, lo cual es un hecho que debe revisarse para Ciudad Victoria puesto que los directores de las empresas tienen estudios universitarios y realizan una planeación estratégica, sin embargo, esto no garantiza la correcta administración de las mismas y, por lo tanto, el éxito competitivo. 
Del mismo modo, es necesario considerar que el sector puede influir en cierta medida en la selección de la estrategia como lo plantea Aragón y Sharma (2003). Esto fue corroborado por Ynzunza e Izar (2013) quienes detectaron que en un estado del centro de México el sector económico modifica la intención estratégica, de manera que las empresas de un sector industrial tienden a aplicar comportamientos estratégicos analizadores y exploradores.

En el caso de nuestra investigación, se denota que posiblemente el sector servicios y el comercial no se ven afectados por las presiones de la industria por lo que tienden a un comportamiento más defensivo. Lo anterior justifica, dentro de los hallazgos, establecer que a este tipo de empresas de la localidad se les dificulte coordinar eficientemente los recursos físicos y humanos, provocando el retraso de su crecimiento

Sin embargo, las conclusiones de esta investigación deben ser interpretadas con limitaciones debido a la poca literatura que estudia el tema estructura familiar y estrategia, lo que no permite generalizar los resultados, los cuales deben tomarse como exploratorios.

\section{5.- REFERENCIAS}

Aguilera, L., y González, M. (2012). Estrategias y rendimiento de las Micro, Pequeñas y Medianas Empresas Familiares. Global Conference of Business and Finance proceedings, 7(1). 877-883.

Alberdi, I., y López, A. (1995). Informe sobre la situación de la familia en España. Ministerio de trabajo y asuntos sociales, Madrid. Consultado el 15 septiembre de 2014. Recuperado de http://www.reis.cis.es/REIS/PDF/REIS_070_141352104264704.pdf.

Aragón, J. (1996). La medición de la estrategia empresarial: Propuesta y validación de una escala multiítem para la medida de la estrategia de negocio según la tipología de Miles y Snow (1978). Cuadernos de Ciencias Económicas y Empresariales, (31), 45-66.

Aragón, J., y Rubio, A. (2005). Factores explicativos del éxito competitivo: el caso de las Pymes del estado de Veracruz. Contaduría y Administración, Facultad de Contaduría y Administración, UNAM, (16) 35-69.

Aragon, J., y Sharma, S. (2003). A contingent resource-based view of proactive corporate environmental strategy. Academy of management review, 28(1), 71-88.

Armenteros M., Elizondo, M., Ballesteros, L., y Molina, V. (2012). Las prácticas de gestión de la innovación en las micro, pequeñas y medianas empresas: resultados del estudio de campo en Piedras Negras Coahuila, México. Revista Internacional Administración y Finanzas, 5(4), 29-50.

Austin, C., Trimm, J., y Sobczak, P. (1995). Information systems and strategic management. Health Care Management Review, 20(3), 26-33. 
Ballina, F., Medina, C., y Martínez, P. (2006). Propuesta del Observatorio Económico para el Desarrollo de la Pequeña y Mediana Empresa en el Estado de Durango. X Congreso Anual de la Academia de Ciencias Administrativas efectuado por la Universidad Autónoma de San Luis Potosí, México.

Beekum, R., y Ginn, G. (1993). Business strategy and interorganizational linkages within the acute care hospitals industry. Human Relations, (46), 129-1317.

Bernal, C. (2010). Metodología de la Investigación. Colombia. Tercera edición. Pearson Educación: Pp. 322.

Camisón, C., Garrigos, F., y Palacios, D. (2007). Estrategias competitivas y desempeño empresarial: estudio comparativo de los modelos de Robinson \& Pearce y Miles \& Snow en el sector hotelero Español. Investigaciones Europeas de Dirección y Economía de la Empresa, 13(3), 161-182.

Castro, E. (2010). Las estrategias competitivas y su importancia en la buena gestión de las empresas. Ciencias Económicas, 28(1), 247-276.

Cermeli, M. (2013). Internacionalización e interacción: estrategias para innovar en las PyMes Vascas. Boletín de Estudios Económicos, LXVIII/208, 147-163.

Cockburn, I., Henderson, R., y Stern, S. (2000). Untangling the origins of competitive advantage. Strategic Management Journal, 21(10/11), 1123-1144.

Conant, J., Mokwa, M., y Varadarajan, P. (1990). Strategic Types, Distinctive Marketing Competencies and Organizational Performance: A Multiple Measures-Based Study. Strategic Management Journal, (11), 365-383.

Cubillo, J. (1997). La inteligencia empresarial en las pequeñas y medianas empresas competitivas de América Latina - algunas reflexiones. Ciencia de la Informacao. Brasilia, 26(3), 260-267.

Daily, C., y Dollinger, M. (1991). Family Firms are Different. Review of Business, 13(1/2), 3-6.

Diario Oficial de la Federación de México (2013, 28 diciembre). REGLAS de Operación del Fondo Nacional Emprendedor para el ejercicio fiscal 2014. [En línea]. Disponible en http://www.dof.gob.mx/nota_detalle.php?codigo=5328349\&fecha=28/12/2013.

Desarbo, W., Di Benedetto, A., Song, M., y Sinha, J. (2005). Revisiting the Miles and Snow strategic framework: Uncovering interrelationships between strategic types, capabilities, environmental uncertainty, and firm performance. Strategic Management Journal, (26), 47-74.

Dvir, D., Segev, E., y Shenhar, A. (1993). Technology's varying impact on the success of strategic business units within the Miles and Snow typology. Strategic Management Journal, (14), 155-162.

García, F. (2007). Estrategia e innovación de la PyMe Industrial en España. Universidad de Cantabria: Ediciones TGD. Pp. 68. 
Gómez, M. (2006). El Futuro de las PYMES en el Marco del TLC. En Rafael Regalado Hernández (Comp.). Las MIPYMES en Latinoamérica. México: Red Latinoamericana de Investigadores en Administración. Consultado en: http://www.eumed.net/libros-gratis/2007b/274/indice4.htm

Grant, R. (1991). The resource based theory of competitive advantage: Implications for strategy formulation. California Management Review, 33(3), 114-135.

Hambrick, D., \& Lei, D. (1985). Toward an empirical prioritization of contingency variables for business strategy. Academy of Management Journal, 28(4), 763-788.

Hambrick, D. (1982). Environmental scanning and organizational strategy. Strategic Management Journal, (3), 159-174.

Hambrick, D. (1983c). Some tests of the effectiveness and functional atributes of Miles and Snow's strategic types. Academy of Management Journal, (26), 5-26.

Hernández, R., Fernández, C., y Baptista, P. (2006). Metodología de la investigación. México: Mc Graw Hill. Pp. 517.

Instituto Nacional de Estadística y Geografía. (2009). Sistema de cuentas nacionales de México. PIB por entidad federativa. Consultado el 10 de Diciembre del 2010. http://www.inegi.org.mx

Instituto Nacional de Estadística y Geografía. (2014). Directorio Estadístico Nacional de Unidades Económicas (DENUE). [En línea].

Consultado en http://www3.inegi.org.mx/sistemas/mapa/denue/default.aspx.

Kauffman S. (2001). El desarrollo de las micro, pequeñas y medianas empresas: un reto para la economía mexicana. Consultado en www. uv.mex/iiesca/revista2001-1/empresas.htm.

Lando, N. (1997). La tipología de estrategias de Miles y Snow: un estudio aplicando una escala multiítems. Revista Europea de Dirección y Economía de la Empresa, (6)2, 33-44.

Lippman, S. y Rumelt, R. (1982). Uncertain imitability: An analysis of interfirm differences in efficiency under competition. Bell Journal of Economics, (13), 218-438.

Maranto, D. (2003). Strategy, distintive competentes and Business performance: a field research in industrial San Luis Potosi, México.

Mccann, J.E., León-Guerrero, A., y Haley, J.D. (2001). Strategic goals and practices of innovative family businesses. Journal of Small Business Management, (39)1, 50-59.

McDaniel, S., y Kolari, J. (1987). Marketing strategy implications of the Miles and Snow strategic tipology. Journal of Marketing, (51), 19-30.

Marroquín, R. (2008). Factores de éxito en dirección estratégica en pequeñas y medianas empresas de la zona metropolitana de Guadalajara. Gesteopolis.com. Recuperado el 10 de septiembre de 2014 de http://www.gestiopolis.com/innovacion-emprendimiento/factores-de-exito-en-direccion-estrategicapymes.htm. 
Miles, R., y Snow, C. (1978). Organizational strategy, structure and process. New York, EE. UU: Mcgraw-Hill. Pp. 271.

Millán (2011). El verdadero Corazón de la economía: las pymes. Consultores Internacionales, S.C. $\begin{array}{lllllll}\text { Consultado el } & 01 & \text { de } & \text { noviembre } & \text { de } & 2013 & \text { en }\end{array}$ http://www.consultoresinternacionales.com/publicaciones/ciscomentario/535/CISComentarioNo535 .pdf.

Münch, L., y Ángeles, E. (2007). Métodos y Técnicas de Investigación. México: Trillas. Pp. 166.

Palomo, M. A. (2005). Los procesos de gestión y la problemática de las PyMes. Ingenierías, (VIII)28, 25 31.

Porter, M. (1980). Competitive Strategy. New York, EE. UU: Free Press. Pp. 432.

Porter, M. (1990). The competitive advantage of nations, New York, The Free Press Word Economic Forum. Traducción (1991): La ventaja competitiva de las naciones. Barcelona, España: Plaza \& Janes. Pp. 857.

Porter, M. (2002). Ventaja competitiva. México: Patria. Pp. 550.

Reed, R., y DeFilippi, R. (1990). Causal ambiguity, barriers to imitation, and sustainable competitive advantage. Academy of Management Review, (15)1, 88-102.

Sánchez, A., Bañón, A., Jiménez, A., y Sangeado, J. (2010). Estrategia y competitividad empresarial: un estudio en las mipymes de Tabasco. Investigación y Ciencia, 18(47), 4-12.

Sánchez, S. (2003). ¿Por qué mueren las empresas? Periódico AM de León. Recuperado de www.leon.uia.mx/publicaciones/laiberoopina23.htm.

Sánchez, Y., Zerón, M., y Mendoza, G. (2014). Análisis de la tipología de estrategias en las pymes de Cd. Victoria Tamaulipas. En Zárate R., Galván, J., Manrique E., y Ramírez M. (ed.), Administración, gestión de la innovación y desarrollo sustentable, México: Universidad Autónoma de Baja California, $1^{\mathrm{a}}$ ed.

Segev, E. (1989). A systematic comparative analysis and synthesis of two business-level strategic typologies. Strategic Management Journal, (10), 487-505.

Shortell, S., y Zajac. E. (1990). Perceptual and Archival Measures of Miles and Snow's Strategic Types: A Comprehensive Assessment of Reliability and Validity. Academy of Management Journal, 4(33), 817-832.

Segev, E., y Weber, Y. (1991). Strategic types and the planning - performance relationships. Strategic Management Journal, (14), 155-162.

Shanker, A., y Astrachan, J. (1996). Myths and realities: Family businesses' contribution to the U.S. economy. A framework for assessing family business statistics. Family Business Review, (9)2, 123167. 
Tan H., López-Acevedo G., Flores R., Rubio S., Slota E., Tinajero M., Beker G. (2007). Evaluando los programas de apoyo a las pequeñas y medianas empresas en México, Banco Mundial, Washington. Pp. 64.

Walker, O.C., y Ruekert, R.W. (1987). Marketing's role in the implementation of business strategies: A critical review and conceptual framework. Journal of marketing, (15) 15-33.

Van Gils, A., Voordecker, W., y Van den Heuvel, J. (2004). Environmental Uncertainty and Strategic Behavior in Belgian Family Firms. European Management Journal, 5(22), 588-595.

Mccann, J.E., Leon-Guerrero, A., y Haley, J.D. (2001). Strategic goals and practices of innovative family businesses. Journal of Small Business Management, 1(39), 50-59.

Veliyath, R., y Shortell, S.M. (1993). Strategic orientation, strategic planning system characteristics and performance. Journal of Management Studies, 3(30), 359-382.

Ynzunza, C.B., y Izar, J.M. (2013). Efecto de las estrategias competitivas y los recursos y capacidades orientados al mercado sobre el crecimiento de las organizaciones. Revista de Contaduría y Administración [online], 1(58), 169-197.

Zahra, S.A., y Pearce, J.A. (1990). Research evidence on the Miles-Snow typology. Journal of Management, (16), 751-768. 